ISSN 0103-5150

Fisioter. Mov., Curitiba, v. 26, n. 3, p. 667-675, jul./set. 2013

Licenciado sob uma Licença Creative Commons

\title{
Fortalecimento dos estabilizadores da cintura escapular na dor no ombro: revisão sistemática
}

\author{
Role of scapular stabilizers strengthening in the \\ painful shoulder: a systematic review
}

Eduardo Luiz Stapait ${ }^{[a]}$, Maira Dalsoglio ${ }^{[b]}$, Angela Marisa Ehlers ${ }^{[b]}$, Gilmar Moraes Santos ${ }^{[c]}$

[a] Mestrando em Fisioterapia na Universidade do Estado de Santa Catarina (Udesc), professor do curso de Fisioterapia da Universidade do Oeste de Santa Catarina (Unoesc), Joaçaba, SC - Brasil, e-mail: eduardo.stapait@gmail.com

[b] Fisioterapeutas pela Universidade do Oeste de Santa Catarina (Unoesc), Joaçaba, SC - Brasil, e-mails: maira_dalsoglio@hotmail.com, angela_ehlers@yahoo.com.br

[c] Doutor em Fisioterapia pela Universidade Federal de São Carlos (UFSCar), professor e coordenador do Programa de Mestrado em Fisioterapia da Universidade do Estado e Santa Catarina (Udesc), Florianópolis, SC - Brasil, e-mail: gilmar.santos@udesc.br

\section{Resumo}

Introdução: 0 controle e a ativação dos músculos estabilizadores são importantes para evitar a dor no ombro; dessa forma, o fortalecimento desses músculos pode auxiliar na redução e prevenção da dor no ombro. Objetivo: Revisar sistematicamente a literatura sobre o efeito do fortalecimento da musculatura estabilizadora da cintura escapular na redução da dor não diagnosticada no ombro. Materiais e métodos: Selecionaram-se ensaios clínicos aleatórios publicados nas bases de dados PubMed, PEDro e Cochrane Library que investigaram o efeito do fortalecimento da musculatura estabilizadora da cintura escapular na dor, função, amplitude de movimento e força muscular de sujeitos adultos ( $\geq 21$ anos) com dor no ombro. A Escala PEDro foi utilizada para determinar a qualidade metodológica dos estudos. Resultados: Após seleção, encontraram-se 489 artigos; destes, cinco artigos corresponderam aos critérios de elegibilidade. Os cinco estudos analisavam o efeito do fortalecimento da musculatura estabilizadora associado a alongamento e outras formas de tratamento. Todos os estudos observaram redução da dor e melhora da função após o fortalecimento associado ao alongamento. A ADM e a força parecem melhorar após o fortalecimento associado ao alongamento. Entretanto, a qualidade metodológica e o número reduzido de estudos para cada desfecho não permitem inferir sobre a efetividade da intervenção. 
Conclusão: $\mathrm{O}$ fortalecimento dos estabilizadores associado ao alongamento diminui a dor e melhora a função do ombro. Futuros ensaios clínicos randomizados devem focar na melhor qualidade metodológica para verificar a efetividade do fortalecimento da musculatura estabilizadora da cintura escapular.

Palavras-chave: Ombro. Dor de ombro. Treinamento de resistência. Técnicas de exercício e de movimento.

\section{Abstract}

Introduction: Control and activation of stabilizer muscles are important to prevent shoulder pain; therefore, strengthening of these muscles could to reduce and to prevent shoulder pain. Objective: To review systematically the literature on the effect of strengthening stabilizing muscles of the shoulder girdle in pain decrease on shoulder undiagnosed pain. Materials and methods: We selected randomized control trials published in PubMed, PEDro and Cochrane Library which investigated the effect of strengthening stabilizing muscles of the shoulder girdle in pain, function, range of motion and muscle strength in adult subjects ( $\geq 21$ years) with shoulder pain. The PEDro scale was used to determine the methodological quality of studies. Results: After selection, were found 489 articles; among them, five articles had corresponded to the eligibility criteria. The five studies examined the effect of strengthening stabilizing muscles associated with stretching and other forms of treatment. All studies reported reduced pain and improved function after the strengthening associated with stretching. ROM and strength seem to improve after the strengthening associated with stretching, however, the methodological quality and small number of study for each outcome, does not allow inferences about the effectiveness of the intervention. Conclusion: The strengthening of stabilizer muscles associated with stretching decreases pain and improves shoulder function. Future randomized control trials should focus on better quality studies to assess the effectiveness of strengthening stabilizing muscles of the shoulder girdle.

Keywords: Shoulder. Shoulder pain. Resistance training. Exercise movement techniques.

\section{Introdução}

A dor no ombro é umas das queixas mais comuns na população em geral, com uma prevalência estimada de $22,3 \%$, afetando principalmente mulheres acima de 50 anos (1). Fatores como flexão ou abdução dos ombros por tempo prolongado, vibrações, postura estática ou carga no membro superior (2), posturas incorretas, uso excessivo do membro superior, práticas de esportes de impacto e alterações anatômicas preexistentes (3) aumentam a probabilidade para que o indivíduo desenvolva dor no ombro (2).

Entre as possíveis causas de dor no ombro, destaca-se a fraqueza e incoordenação dos músculos estabilizadores da cintura escapular. 0 controle e ativação desses músculos são fundamentais para as atividades que envolvem o membro superior. Alterações do grupo muscular estabilizador parecem estar associadas às disfunções da cintura escapular (4-6). Dentre os músculos estabilizadores, destacam-se manguito rotador, trapézio inferior e trapézio médio $(4,7-10)$.

Como tratamento da dor no ombro, a fisioterapia é a conduta mais adequada para restabelecer o equilíbrio muscular e melhorar a funcionalidade do membro acometido. Existem várias modalidades fisioterapêuticas utilizadas para o tratamento da dor no ombro, tais como terapia manual, eletroterapia e terapia com exercícios (11-13).

Recentemente, exercícios que focam a estabilização da cintura escapular são utilizados por fisioterapeutas como forma de tratamento das dores no ombro. 0 tratamento tem como base corrigir os padrões de movimento e recrutamento muscular, trabalhando com exercícios para restabelecer a dinâmica dos músculos (14), respeitando as capacidades musculares normais dos indivíduos para o planejamento de um programa de exercícios (15).

A combinação desses exercícios com a terapia manual parece eficaz tanto na redução da dor como na melhora da função em todas as condições que produzam dor no ombro (13). Apesar dos possíveis benefícios desses exercícios, ainda faltam estudos que estabeleçam consenso sobre os tratamentos, desfechos clínicos esperados e que orientem a utilização na prática clínica. Portanto, o objetivo deste estudo foi realizar uma revisão sistemática para investigar a efetividade 
do fortalecimento da musculatura estabilizadora da cintura escapular na diminuição da dor e melhora da função da articulação do ombro em adultos.

\section{Materiais e métodos}

\section{Critérios de elegibilidade}

Incluíram-se nesta revisão ensaios clínicos aleatórios que trouxeram conteúdo sobre o efeito do fortalecimento dos músculos estabilizadores da cintura escapular, realizados em adultos ( $\geq 21$ anos). Os desfechos clínicos analisados foram dor, função do ombro, amplitude de movimento (ADM) e força. Não foi adotado nenhum limite para período de publicação; os idiomas analisados foram inglês e português.

0 fortalecimento dos músculos estabilizadores da cintura escapular foi analisado exclusivamente ou em associação com outra técnica nas comparações com: grupo controle; tratamento medicamentoso; tratamento biopsicossocial e educação; placebo; supervisão de profissional e falta desta. Foram excluídos da revisão os estudos que não relataram com clareza a intervenção, tratamentos realizados após procedimentos cirúrgicos e estudos não reportados na língua inglesa e portuguesa.

\section{Estratégias de busca}

A coleta de dados foi realizada nas bases de dados PubMed (National Library of Medicine e National Institutes of Health), PEDro (Physiotherapy Evidence Database), e Cochrane Library.

Os seguintes termos foram utilizados como descritores de busca: shoulder, shoulder pain, physical therapy, muscle weakness, scapula, muscle tonus, muscle fatigue, stabilization. Para o cruzamento dos descritores usados na busca, foram empregadas a expressão "booleana" que compreende os códigos "AND" (intercessão de dois ou mais assuntos), "OR" (localização individual dos assuntos e/ou a soma de dois ou mais assuntos), respeitando-se as especificidades de cada base de dados.

\section{Seleção dos estudos}

Após a busca nas bases de dados, foi realizada a seleção dos artigos pelos títulos e resumos. Dois revisores de forma independente fizeram a leitura dos títulos e resumos; discordâncias foram resolvidas por consenso. Caso não fosse possível a identificação pelo resumo, o artigo era avaliado na íntegra. Uma segunda busca foi realizada nas referências dos estudos encontrados na primeira busca, a fim de detectar estudos que não tivessem sido identificados na busca inicial. Estudos que se enquadraram nos critérios de elegibilidade foram selecionados.

\section{Avaliação da qualidade metodológica}

Para avaliação da qualidade metodológica, utilizou-se a escala PEDro em português (16). A escala PEDro consiste em 11 itens com opções de resposta "Sim" e "Não". A somatória de respostas "sim" constitui o escore final. É um instrumento validado e apresenta boa confiabilidade na mensuração da qualidade metodológica de estudos clínicos randomizados (17-20). Dois revisores aplicaram de forma individual os critérios da escala; em caso de controvérsia, um terceiro avaliador foi consultado para que em consenso fosse atribuído ou não o critério duvidoso.

\section{Resultados}

Na busca realizada em maio de 2011, foram encontrados 605 potenciais estudos nas bases de dados consultadas. Destes, apenas cinco artigos respeitaram os critérios de elegibilidade e foram incluídos na revisão (Figura 1). 0 número total de sujeitos incluídos nos estudos variou de 30 a 138, totalizando 356 sujeitos avaliados.

\section{Avaliação da qualidade metodológica}

Estudos com alta qualidade metodológica fornecem maior evidência sobre a eficácia dos exercícios de fortalecimento dos estabilizadores da cintura escapular para tratamento de indivíduos com dor no ombro. A pontuação dos estudos na Escala PEDro obteve uma média de 5,8 para um total de 10 pontos. Dos cinco estudos incluídos, um apresentou baixa qualidade metodológica (21) (PEDro 4/10); três, qualidade moderada (22-24) (PEDro 6/10); um, boa qualidade (25) (PEDro 7/10).

Várias falhas metodológicas foram reconhecidas entre os estudos, incluindo falha de alocação dos 


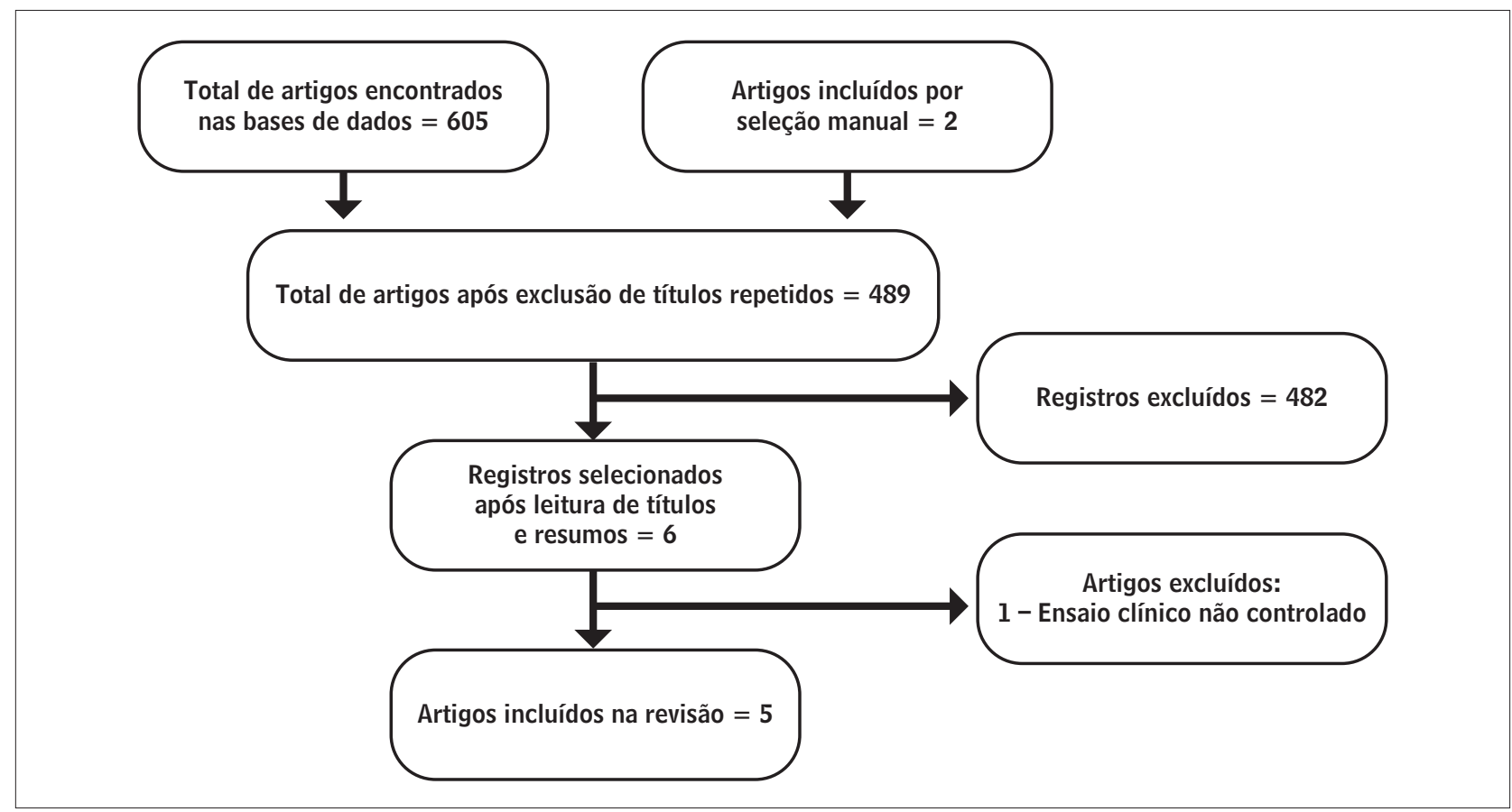

Figura 1 - Fluxograma do processo de seleção dos estudos Fonte: Dados da pesquisa.

sujeitos, diferença dos grupos no início e final do estudo, falha de mascaramento dos avaliadores, análise do tratamento por pelo menos um resultado-chave e falha no mascaramento de terapeutas e indivíduos. 0 percentual de estudos que satisfez cada pontuação metodológica da Escala PEDro está especificada na Tabela 1. As características dos estudos incluídos estão sumarizadas no Quadro 1.

\section{Análise dos desfechos}

\section{Dor}

Os cinco estudos (21-25) avaliaram a dor por meio da Escala Visual Analógica (EVA). Todos os estudos mostraram redução da dor para o grupo que utilizou os exercícios de estabilização. No estudo realizado por Ginn et al. (25), não houve apresentação numérica dos resultados, mas os autores concluíram que ocorreu redução significativa da dor e aumento da independência com cuidados pessoais diários nos indivíduos que realizaram o tratamento durante 30 dias. Já para Senbursa et al. (21), ambos os grupos apresentaram redução da dor; porém, o grupo que realizou terapia manual associada a alongamentos, fortalecimentos da musculatura estabilizadora da escápula e crioterapia (GTM) os resultados evidenciaram maior nível de redução média $(70,1 \%)$ no quadro doloroso do que no grupo que realizou fortalecimento associados com alongamentos $(54,5 \%)$.

Para Wang e Trudelle-Jackson (22), não houve diferença significativa, após 8 semanas de tratamento, na redução da dor entre os grupos. 0 grupo que realizou exercícios específicos para os estabilizadores da cintura escapular obteve redução média de 54,3\%, e o grupo que realizou exercícios não específicos para os estabilizadores da cintura escapular, com exceção do fortalecimento dos rotadores internos e externos obteve redução média de $56,1 \%$. No estudo realizado por Ludewig e Borstad (23), os resultados foram clinicamente menores, mas houve redução da dor. 0 grupo intervenção apresentou melhora significativa da dor em relação ao grupo controle, com uma redução média de pré-teste para pós-teste de 41,6\%; os grupos controle e assintomático apresentaram uma redução de $10,8 \%$ e $7,6 \%$, respectivamente.

Maior redução da dor foi observada no estudo conduzido por Ginn e Cohen (24). Após cinco semanas, todos os grupos obtiveram resultados significativos para redução da dor e melhora da 
Tabela 1 - Número e porcentagem dos critérios da Escala PEDro

\begin{tabular}{lcc}
\hline Escala PEDro & $\begin{array}{c}\text { Estudos que seguiram os } \\
\text { critérios da Escala }\end{array}$ & $\begin{array}{c}\text { Porcentagem dos artigos que } \\
\text { seguiram os critérios da Escala (\%) }\end{array}$ \\
\hline 1 - Critérios de elegibilidade & 5 & 100 \\
2 - Aleatória dos sujeitos & 5 & 100 \\
3 - Alocação oculta dos sujeitos & 3 & 60 \\
4 - Grupos semelhantes no início do estudo & 4 & 80 \\
5 - Sujeitos cegos & 0 & 0 \\
6 - Terapeutas cegos & 0 & 0 \\
7 - Avaliadores cegos & 3 & 60 \\
8 - Mensuração resultado-chave (85\%) & 3 & 60 \\
9 - Intenção de tratamento & 1 & 20 \\
10 - Comparação entre os grupos & 5 & 100 \\
11 - Medidas de precisão e variabilidade & 5 & 100 \\
\hline
\end{tabular}

Fonte: Dados da pesquisa.

função. No grupo que realizou exercícios, houve uma redução de $80 \%$ após cinco semanas, no grupo injeção uma redução de $89 \%$ e no grupo que realizou diversas modalidades terapêuticas houve uma redução de $61 \%$.

\section{Desempenho funcional}

Os cinco estudos avaliaram a melhora da função do ombro realizando exercícios de estabilização associados ao alongamento muscular da cintura escapular. No estudo de Ginn e Cohen (24), a terapia por exercício destinada a restabelecer os mecanismos de estabilização e coordenação muscular foram eficazes, em curto prazo, na melhora da função. Para Senbursa et al. (21), os dois grupos avaliados em seu estudo obtiveram bons resultados quanto à melhora da função, mas o grupo GTM apresentou resultados mais significativos. No estudo realizado por Wang e Trudelle-Jackson (22), os resultados não suportam a hipótese de que os exercícios específicos para os estabilizadores da cintura escapular seriam mais eficazes que os exercícios não específicos. Esses autores não observaram diferença significativa entre indivíduos de ambos os grupos no desempenho funcional.
Amplitude de movimento (ADM)

Em relação à amplitude de movimento, dois estudos evidenciaram melhores resultados quando os indivíduos realizaram exercícios de fortalecimento associados a técnicas de alongamento. No estudo realizado por Wang e Trudelle-Jackson (22), ambos os grupos tiveram melhora significativa da ADM para o movimento de abdução. Para o grupo que realizou exercícios específicos para os estabilizadores da cintura escapular, a abdução de ombro aumentou em $11,8 \%$ após oito semanas de tratamento. Ginn e Cohen (24) avaliaram apenas os movimentos de abdução e flexão de ombro após cinco semanas de tratamento. Houve aumento da ADM sem dor e total da ADM para abdução e flexão em todos os grupos avaliados, sem diferenças entre os grupos. Vale ressaltar que nos dois estudos, apesar da melhora estatística, os resultados não apresentam mudanças clínicas significativas, pois o ganho na ADM não foi relevante funcionalmente.

\section{Força muscular}

Dois estudos investigaram a força muscular do complexo do ombro. Para Wang e Trudelle-Jackson 
Quadro 1 - Características dos estudos incluídos

\begin{tabular}{|c|c|c|c|c|}
\hline Estudo & Participantes & Desfechos & Resultados & PEDro \\
\hline $\begin{array}{l}\text { Ginn et al. } \\
(25)\end{array}$ & $\begin{array}{l}\text { n = } 66 \text { (GC e GT não especificam o número de } \\
\text { participantes de cada grupo) } \\
\text { GC - sem tratamento } \\
\text { GT - fortalecimento com objetivo de restaurar a } \\
\text { estabilidade da cintura escapular }\end{array}$ & $\begin{array}{l}\text { Percepção da } \\
\text { melhora } \\
\text { Dor - EVA } \\
\text { ADM sem dor }\end{array}$ & $\begin{array}{l}0 \text { GT apresentou melhora em todos } \\
\text { os desfechos, a diminuição da dor } \\
\text { não apresentou diferença entre os } \\
\text { grupos. }\end{array}$ & $7 / 10$ \\
\hline $\begin{array}{l}\text { Senbursa, } \\
\text { Baltaci e } \\
\text { Atay (21) }\end{array}$ & $\begin{array}{l}\text { GE }(n=15) \text { - alongamentos e fortalecimentos dos } \\
\text { músculos estabilizadores, realizados em domićílio } \\
\text { GTM }(n=15) \text { - mobilização, aplicação de gelo, } \\
\text { alongamentos e fortalecimentos do complexo do } \\
\text { ombro }\end{array}$ & $\begin{array}{l}\text { Dor - EVA } \\
\text { ADM } \\
\text { Questionário de } \\
\text { NEER }\end{array}$ & $\begin{array}{l}\text { Os dois grupos apresentaram } \\
\text { melhora nos desfechos dor e } \\
\text { questionário de NEER; o GTM } \\
\text { aumentou significativamente a ADM } \\
\text { flexão, adução e rotação externa. Na } \\
\text { comparação entre os grupos, o GTM } \\
\text { obteve melhores resultados. }\end{array}$ & $4 / 10$ \\
\hline $\begin{array}{l}\text { Wang e } \\
\text { Trudelle- } \\
\text { Jackson } \\
\text { (22) }\end{array}$ & $\begin{array}{l}\text { GEPersonalizado }(n=15) \text { - autoalongamento, } \\
\text { fortalecimento personalizados para estabilizadores } \\
\text { do ombro } \\
\text { GEPadrão }(n=15) \text { - fortalecimento padrão para } 0 \\
\text { complexo do ombro }\end{array}$ & $\begin{array}{l}\text { Dor - EVA } \\
\text { ADM } \\
\text { Desempenho } \\
\text { funcional - } \\
\text { Flexilevel Scale } \\
\text { of Shoulder } \\
\text { Function } \\
\text { Força muscular } \\
\text { (dinamômetro) }\end{array}$ & $\begin{array}{l}\text { Não houve diferença entre os grupos } \\
\text { para os desfechos analisados. }\end{array}$ & $6 / 10$ \\
\hline $\begin{array}{l}\text { Ludewig } \\
\text { e Borstad } \\
\text { (23) }\end{array}$ & $\begin{array}{l}\text { GT }(n=34) \text { alongamento e fortalecimento do ombro } \\
\text { GTSintomático }(n=33) \text { não realizaram tratamento } \\
\text { GTAssintomático }(n=25) \text { não realizaram tratamento }\end{array}$ & $\begin{array}{l}\text { Dor - EVA } \\
\text { Incapacidade } \\
\text { Shoulder Rating } \\
\text { Questionnaire } \\
\text { (SRQ) }\end{array}$ & $\begin{array}{l}0 \text { GT apresentou melhores resultados } \\
\text { em todos os desfechos analisados } \\
\text { em comparação ao GTSintomático. }\end{array}$ & $6 / 10$ \\
\hline $\begin{array}{l}\text { Ginn e } \\
\text { Cohen } \\
\text { (24) }\end{array}$ & $\begin{array}{l}\text { PR }(n=77) \text { - apresentavam redução da ADM para } \\
\text { flexão e/ou abdução } \\
\text { R ( } n=61) \text { - não apresentaram redução da ADM } \\
\text { População posteriormente dividida em: } \\
\text { GE }(n=48) \text { alongamento, fortalecimento e } \\
\text { restauração do ritmo escapuloumeral } \\
\text { GI }(n=48) \text { - aplicação de corticosteroide } \\
\text { GMPM ( } n=42) \text { combinação de eletro/exercícios } \\
\text { para ADM e força }\end{array}$ & $\begin{array}{l}\text { Dor - EVA } \\
\text { Função } \\
\text { (pontuação } \\
\text { autorrelatada) } \\
\text { Força isométrica } \\
\text { (dinamômetro) }\end{array}$ & $\begin{array}{l}\text { Todos apresentaram melhora nos } \\
\text { desfechos analisados, sem diferenças } \\
\text { entre os grupos. }\end{array}$ & $6 / 10$ \\
\hline
\end{tabular}

Fonte: Dados da pesquisa.

(22), tanto o grupo que realizou exercícios não específicos para os estabilizadores da cintura escapular quanto o grupo que realizou exercícios específicos para os estabilizadores da cintura escapular obtiveram maior força muscular para movimento de abdução do ombro (22,7\%), maior força dos rotadores internos (21\%) e externos (20,5\%), de trapézio médio $(37,8 \%)$ e trapézio inferior (44\%) após oito semanas de tratamento. Ginn e Cohen (24) avaliaram a força apenas para o movimento de abdução de ombro, observando maior força em todos os grupos, tanto no grupo que realizou fortalecimento para os estabilizadores $(17,1 \%)$, quanto no grupo que recebeu injeção $(18,1 \%)$ e no grupo que recebeu diversas modalidades terapêuticas $(18,1 \%)$.

\section{Fortalecimento dos estabilizadores} sem supervisão

Dois estudos compararam um programa de fisioterapia individualizado, realizado sem supervisão pelos pacientes em suas residências com orientação inicial de um fisioterapeuta. No estudo realizado por et al. (23), em trabalhadores de construção civil, os resultados sugerem que um programa de exercícios 
em casa pode ser eficaz na redução dos sintomas e melhora da função nesses trabalhadores com dor no ombro. Contudo, no estudo realizado por Senbursa, Baltaci e Atay (21), as técnicas de terapia manual com fortalecimento dos estabilizadores e alongamentos, associadas a aplicação de gelo e mobilizações realizadas por um fisioterapeuta na clínica apresentaram melhores resultados para redução da dor, melhora da função e aumento da força muscular quando comparado ao grupo que realizou exercícios domiciliares.

\section{Discussão}

\section{Avaliação da qualidade metodológica}

Estudos com alta qualidade metodológica fornecem maior evidência quanto à eficácia da estabilização da cintura escapular em sujeitos com dor no ombro, justificando sua utilização no ambiente clínico. Entretanto, deve-se reconhecer que estudos que mostram resultados favoráveis não fornecem necessariamente a maior evidência se apresentarem uma baixa qualidade metodológica. A pontuação na Escala PEDro dos cinco estudos incluídos nesta revisão alcançaram média de 5,8 para um total de 10 pontos. Artigos de alta qualidade metodológica devem satisfazer mais de $70 \%$ dos critérios da escala (20). Nesta revisão, apenas um artigo pode ser classificado com alta qualidade metodológica (25).

0 fracasso da maioria dos estudos em atender aos critérios da qualidade metodológica impacta significativamente na validade das provas a serem abstraídas. Os vieses encontrados nos estudos selecionados foram: falha de mascaramento dos avaliadores $(21,23)$, inadequada medida dos resultados-chave $(21,22)$, falta de ocultação da alocação dos sujeitos $(21,24)$ e intenção de tratamento $(21,22,24,25)$.

0 mascaramento dos avaliadores é importante para evitar influência no tratamento, a fim de garantir uma medida acurada do efeito de uma determinada intervenção. A avaliação cega produz resultados mais consistentes do que as avaliações sem esse mascaramento (26). A ausência de um acompanhamento adequado torna difícil confirmar a efetividade das técnicas aplicadas, pois isso significa que os avaliadores não acompanharam a evolução dos indivíduos para analisar se suas metas foram alcançadas. A forma de randomização deve estar clara, pois a falha na ocultação da alocação dos sujeitos pode interferir nos resultados do estudo.

\section{Redução da dor}

Houve redução da dor em todos os estudos analisados. Quando realizados exercícios de fortalecimento dos estabilizadores da cintura escapular e alongamentos em casa houve redução da dor $(21,23)$. A associação do fortalecimento com técnicas de terapia manual, aplicação de gelo e mobilizações realizadas por um fisioterapeuta na clínica reduziram significativamente a dor e foram superiores ao fortalecimento associado ao alongamento (21). Há evidências de que a estabilização associada ao alongamento reduz a dor (21-25), entretanto, evidências para associação de outras técnicas com a estabilização, como eletroterapia (24), mobilizações com aplicação de gelo (21), não podem ser sugeridas devido à baixa qualidade metodológica e o número reduzido de estudos. A realização de exercícios específicos para os estabilizadores da cintura escapular parece não exercer influência na redução da dor (22).

A injeção de corticosteroide apresenta os mesmos benefícios dos exercícios de estabilização (24). Essa afirmação vem ao encontro ao estudo de Crawshaw et al. (27), que não observou diferença significativa na dor no ombro em participantes que receberam uma injeção associada a exercícios em comparação com aqueles que receberam apenas exercícios. Sabe-se que o alívio da dor no início do tratamento é uma prioridade e pode ser alcançada rapidamente com o uso de injeção (28), mas exercícios fisioterápicos parecem ser a melhor opção para os indivíduos tanto a curto como a longo prazo $(24,29)$.

\section{Desempenho funcional}

Assim como a dor, a função também apresentou resultados positivos, com melhora em todos os estudos analisados. $\mathrm{O}$ fortalecimento dos estabilizadores da cintura escapular e alongamentos associados à aplicação de gelo e mobilizações realizadas por um fisioterapeuta na clínica (21) apresentaram melhora da função do ombro e foram mais eficazes do que apenas exercícios de estabilização e alongamentos realizados em casa $(21,23)$. Exercícios de estabilização e alongamentos associados ao uso de eletroterapia (24) e também a 
mobilizações com aplicação de gelo (21) se mostram eficazes na melhora da função do ombro; porém, essas técnicas não se mostram superiores a exercícios de estabilização associados apenas com alongamentos. Assim como na dor, a realização de exercícios específicos para os estabilizadores da cintura escapular parece não exercer influência na melhora da função (22).

\section{Amplitude de movimento}

Dois estudos apresentaram resultados em relação à amplitude de movimento $(22,25)$. 0 estudo conduzido por Wang e Trudelle-Jackson (22) observou aumento médio de $16,1^{\circ}$ no movimento de abdução e Ginn et al. (25) relataram aumento médio de $16^{\circ}$ na ADM sem dor para o movimento de flexão e $22^{\circ}$ para o movimento de abdução. Esses resultados não implicam em mudanças clínicas e funcionais, apresentando aumento de poucos graus em relação à amplitude funcional para os membros superiores.

\section{Força muscular}

Evidências limitadas indicam os exercícios de estabilização para o aumento da força dos músculos da cintura escapular. Entretanto, essa melhora pode estar associada à redução do quadro álgico na região. Permanecendo incerta a efetividade dos exercícios de estabilização nessa variável.

\section{Limitação do estudo}

A restrição apenas aos idiomas inglês e português e a utilização de três bases de dados limitam a seleção dos estudos, tornando possível omitir outros estudos com boa qualidade metodológica escritos em outros idiomas e indexados em bases de dados não incluídas nesta revisão. Além disso, a heterogeneidade dos estudos incluídos não permitiu uma discussão clara quanto a amostra, duração dos sintomas, duração do tratamento e técnica empregada.

\section{Conclusão}

O fortalecimento dos estabilizadores da cintura escapular associado ao alongamento diminui a dor e melhora a função do ombro. Não se pode fazer inferências sobre a associação com outras técnicas e acompanhamento na execução dos exercícios devido à escassez de estudos e à baixa qualidade metodológica dos estudos analisados. Futuros ensaios clínicos randomizados devem focar na melhor qualidade metodológica e homogeneidade das amostras e instrumentos de avaliação para que novas revisões sistemáticas possam determinar com maior convicção a efetividade dos exercícios de estabilização na redução da dor, melhora da incapacidade e restauração da $\mathrm{ADM}$ de sujeitos com dor no ombro.

\section{Referências}

1. Hill CL, Gill TK, Shanahan EM, Taylor AW. Prevalence and correlates of shoulder pain and stiffness in a population-based study: the North West Adelaide Health Study. Int J Rheum Dis. 2010;13(3):215-22.

2. De Mendonça HP Junior, Assunção AA. Associação entre distúrbios do ombro e trabalho: breve revisão da literatura. Rev Bras Epidemiol. 2005;8(2):167-76.

3. Facci LM. Síndromes dolorosas do ombro: análise de sua incidência e características. Arq Ciências Saúde UNIPAR. 2000;4(3):195-200.

4. Illyés A, Kiss RM. Kinematic and muscle activity characteristics of multidirectional shoulder joint instability during elevation. Knee Surg Sports. Traumatol Arthrosc. 2006;14(7):673-85.

5. Ludewig PM, Cook TM. Alterations in shoulder kinematics and associated muscle activity in people with symptoms of shoulder impingement. Phys Ther. 2000;80(3):276-91.

6. Smith M, Sparkes V, Busse M, Enright S. Upper and lower trapezius muscle activity in subjects with subacromial impingement symptoms: is there imbalance and can taping change it?. Phys Ther Sport. 2009;10(2):45-50.

7. Merolla G, De Santis E, Campi F, Paladini P, Porcellini G. Supraspinatus and infraspinatus weakness in overhead athletes with scapular dyskinesis: strength assessment before and after restoration of scapular musculature balance. Musculoskelet Surg. 2010;94(3):119-25.

8. Kelly BT, Williams RJ, Cordasco FA, Backus SI, Otis JC, Weiland DE, et al. Differential patterns of muscle activation in patients with symptomatic and asymptomatic rotator cuff tears. J Shoulder Elbow Surg. 2005;14(2):165-71. 
9. Celik D, Sirmen B, Demirhan M. The relationship of muscle strength and pain in subacromial impingement syndrome. Acta Orthop Traumatol Turc. 2011;45(2):79-84.

10. Phadke V, Camargo PR, Ludewig PM. Scapular and rotator cuff muscle activity during arm elevation: a review of normal function and alterations with shoulder impingement. Rev Bras Fisioter. 2009;13(1):1-9.

11. Brantingham JW, Cassa TK, Bonnefin D, Jensen M, Globe G, Hicks M, et al. Manipulative therapy for shoulder pain and disorders: expansion of a systematic review. J Manipulative Physiol Ther. 2011;34(5):314-46.

12. Ho CY, Sole G, Munn J. The effectiveness of manual therapy in the management of musculoskeletal disorders of the shoulder: a systematic review. Man Ther. 2009;14(5):463-74.

13. Marinko LN, Chacko JM, Dalton D, Chacko CC. The effectiveness of therapeutic exercise for painful shoulder conditions: a meta-analysis. J Shoulder Elbow Surg. 2011;20(8):1351-9.

14. Magarey ME, Jones MA. Dynamic evaluation and early management of altered motor control around the shoulder complex. Man Ther. 2003;8(4):195-206.

15. Phadke V, Camargo P, Ludewig P. Scapular and rotator cuff muscle activity during arm elevation: a review of normal function and alterations with shoulder impingement. Rev Bras Fisioter. 2009;13(1):1-9.

16. Shiwa SR, Costa LO, Costa LC, Moseley AM, Hespanhol LC Júnior, Venâncio R, et al. Reproducibility of the Portuguese version of the PEDro Scale. Cad Saúde Pública. 2011;27(10):2063-8.

17. de Morton NA. The PEDro scale is a valid measure of the methodological quality of clinical trials: a demographic study. Aust J Physiother. 2009;55(2):129-33.

18. Bhogal SK, Teasell RW, Foley NC, Speechley MR. The PEDro scale provides a more comprehensive measure of methodological quality than the Jadad scale in stroke rehabilitation literature. J Clin Epidemiol. 2005;58(7):668-73.

19. Macedo LG, Elkins MR, Maher CG, Moseley AM, Herbert RD, Sherrington C. There was evidence of convergent and construct validity of Physiotherapy Evidence Database quality scale for physiotherapy trials. J Clin Epidemiol. 2010;63(8):920-5.
20. Maher CG, Sherrington C, Herbert RD, Moseley AM, Elkins M. Reliability of the PEDro scale for rating quality of randomized controlled trials. Phys Ther. 2003;83(8):713-21.

21. Senbursa G, Baltaci G, Atay A. Comparison of conservative treatment with and without manual physical therapy for patients with shoulder impingement syndrome: a prospective, randomized clinical trial. Knee Surg Sports Traumatol Arthrosc. 2007;15(7):915-21.

22. Wang SS, Trudelle-Jackson EJ. Comparison of customized versus standard exercises in rehabilitation of shoulder disorders. Clin Rehabil. 2006;20(8):675-85.

23. Ludewig PM, Borstad JD. Effects of a home exercise programme on shoulder pain and functional status in construction workers. Occup Environ Med. 2003;60(11):841-9.

24. Ginn KA, Cohen ML. Exercise therapy for shoulder pain aimed at restoring neuromuscular control: a randomized comparative clinical trial. J Rehabil Med. 2005;37(2):115-22.

25. Ginn KA, Herbert RD, Khouw W, Lee R. A randomized, controlled clinical trial of a treatment for shoulder pain. Phys Ther. 1997;77(8):802-9

26. Jadad AR, Moore RA, Carroll D, Jenkinson C, Reynolds DJ, Gavaghan DJ, et al. Assessing the quality of reports of randomized clinical trials: is blinding necessary? Control Clin Trials. 1996;17(1):1-12.

27. Crawshaw DP, Helliwell PS, Hensor EMA, Hay EM, Aldous SJ, Conaghan PG. Exercise therapy after corticosteroid injection for moderate to severe shoulder pain: large pragmatic randomised trial. BMJ. 2010;340:c3037.

28. Gialanella B, Prometti P. Effects of corticosteroids injection in rotator cuff tears. Pain Med. 2011;12(10):1559-65.

29. Buchbinder R, Green S, Youd JM. Corticosteroid injections for shoulder pain. Cochrane Database Syst Rev. 2003;(1):CD004016.

Recebido: 27/06/2012

Received: 06/27/2012

Aprovado: $13 / 02 / 2013$

Approved: 02/13/2013 